\title{
The Analysis of Technical Solutions for Medical Ozonators
}

\author{
Sokol E.I., Kipenskiy A.V., Kulichenko V.V., Tomashevskiy R.S., Barkhotkina T.M. \\ Dep. "Industrial and Biomedical Electronics" \\ The National Technical University "Kharkiv Polytechnic Institute" \\ Kharkiv, Ukraine \\ kavkpi@ukr.net
}

\begin{abstract}
The article deals with the basic principles of control systems construction in the medical ozone generators. The authors present and analyzes the specific diagram of the electrical and pneumatic part of ozonator. According to analysis results the authors give recommendations for the considered systems realization in the medical ozone generators.
\end{abstract}

Keywords - ozonator, discharge chamber, control system, flow rate control, ozone concentration.

\section{INTRODUCTION}

Ozone $\left(\mathrm{O}_{3}\right)$ is an allotropic modification of oxygen with a pungent aroma, its molecule consists of three oxygen atoms and has an asymmetric structure of triangle. Ozone is highly soluble in water, it is a strong oxidant and quickly decomposes at ambient temperature. Impact of high concentrations of ozone is dangerous, it leads to the defeat of the respiratory tract, lungs and mucous membranes. Ozone is used in medical practice as an ozone-oxygen mixture (OOM) with different levels of ozone concentrations (OC) in it. The method of using OOM for medicinal purposes is called ozone therapy. Healthiness properties of ozone have been known at the beginning of the last century, but the development of the method has begun only 35-40 years ago. The OOM has a wide range of positive effects on humans; it has bactericidal, antiviral, antifungal, immunomodulatory, antihypoxic, cytostatic, disintoxicational properties. It is high tolerated and side effects are practically absent. Ozone therapy is used in surgery and therapy for eye diseases, urology, obstetrics and gynecology, dermatology, dentistry, and for sexually transmitted infections, diseases of the upper respiratory tracts and lungs, neurology, anesthesiology, pediatrics, reanimation, intensive care and cosmetics. Medical use of OOM shortens treatment time, reduces mortality and disability [1].

However, the use of OOM for medicinal purposes has a high profitability compared to drug treatments because consumable materials are oxygen and small electricity consumption. Methods of ozone therapy can be conventionally divided into three groups depending on the use of OOM: external impact, parenteral methods of exposure, enteral methods. [2].

Each of the methods is characterized not only by a certain sequence of actions, but also by the parameters of OOM, to which OC in OOM and flow of OOM are related [3]. For example, OOM with OC from 2.5 to $100 \mathrm{mg} / 1$, oil - from 5 to $30 \mathrm{mg} / 1$ are used for bubbling the distillate and saline solutions. OOM with a concentration of 1 to $10 \mathrm{mg} / 1$ is used for flow gassing. OOM with a concentration of 10 to $40 \mathrm{mg} / 1$ is used in large and small autohemotherapy. OOM concentration of 5 to $25 \mathrm{mg} / 1$, rectal insufflations from 10 to $60 \mathrm{mg} / 1$ is conducted with subcutaneous injections. The range flow control of OOM is from 0.1 to $21 / \mathrm{min}$ at preparing for the ozone therapy treatments. Therefore, OC in OOM should be regulated in the range of 0.1 to $100 \mathrm{mg} / 1$, i.e. $1: 1000$, and the flow of OOM - from 0.1 to $2.01 / \mathrm{min}$, i.e. $1: 20$ to assurance the implementation of all methods of ozone therapy.

The purpose of this paper is to analyze the various technical solutions of medical ozonators, that allow to regulate the output parameters of the ozone-oxygen mixture.

The principle of operation of the most medical ozonators is based on the receiving of ozone from medical oxygen with the help of electrophysical method that consists in creation of a volume barrier discharge in gas gap of the discharge chamber (DC), to which a high-voltage alternating current is attached. The sources of oxygen for ozonator are balloons with medical oxygen or oxygen network of medical institutions. Because at the output of ozonator there is a necessity to get OOM with two adjustable parameters, it is obvious that such regulation should be carried out in two control systems: electrical control system (ECS) to regulate OC in OOM and pneumatic control system (PCS) to control the flow of OOM. In this case, each system must contain informational part (IP) that collects information, its transformation, processing and presentation. In addition, each control system must be provided for the executive part (EP). In ECS EP provides to convert the parameters (voltage, current, frequency) of electrical energy from a power line, and its distribution. Thus, in the ECS EP manages energy flow. In PCS EP provides control parameters (pressure, flow) of gas coming from the cylinder or oxygen network, i.e. manages the real flow.

\section{ElECTRICAL CONTROL SySTEM}

The analysis of the operation conditions of such control systems has shown that the stability of the OOM formation and its flow rate would affect a number of disturbing factors. With respect to the electrical control system, main factors are the deviation from the nominal voltage value $u_{\mathrm{PS}}$ of power supply, pressure and temperature of OOM in the discharge chamber. In relation to the pneumatic control system - the oxygen pressure 
at the input of ozonator, resistance of its pneumatic channel and the external load.

In the process of regulation of OC in OOM it is necessary to modify the amplitude-frequency-time parameters of alternating voltage $u_{\mathrm{A}}$ at the output of energetic part of the electrical control system [4]. To stabilize OC in OOM with that having disturbing factors, the most simple solution, at first sight, is the solution to use a closed-loop automatic control with the organization of feedback exactly at $\mathrm{OC}$ in OOM (OC sensor $\left(\mathrm{S}_{\mathrm{OC}}\right)$ in Fig. 1). Control signal $u_{\mathrm{C}}$ in this system is formed with the consideration of the setting signal $u_{\mathrm{S}}$ corresponding to $\mathrm{OC}$, and feedback signal $u_{\mathrm{FB}}$ at the output from the $\mathrm{S}_{\mathrm{OC}}$.

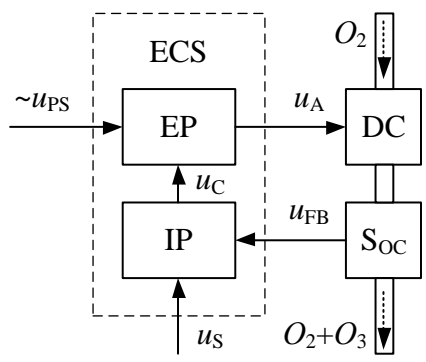

Fig. 1. Structure of ECS with ozone concentration sensor

The analysis of technical means according to the measurement of OC in OOM (optical gas analysis indicators "THE TSIKLON", CJSC "OPTEK", St. Petersburg; photometric meters of ozone concentration "MEDOZONE", "MEDOZONE LTD.", Moscow; optical analyzers of ozone concentration "ICO", JSC "LEPSE" Kirov, etc.), has shown that they mostly use photometric method for the ozone determination on resonance-absorption band at wavelength $\lambda=253.7 \mathrm{~nm}$. This method is justified for measuring devices. However, it use in automatic control systems in real-time, it makes some difficulties. Firstly, it is inertia, and secondly - the need for periodic gas supply, which does not contain ozone in the optical cell to calibrate measuring means, and thirdly - the availability of sufficiently enough bulky resonant source of ultraviolet radiation with high temporal stability of power and wavelength $\lambda=253.7 \mathrm{~nm}$.

Another, equally effective approach to the problem of stabilization of OC in OOM is the fact that the electrical system control provides compensation for disturbing factors impact. Of all the factors that have the greatest influence on the ozone formation, the most significant is the instability of voltage of power line [5].

In order to ensure the control alternating voltage on the discharge chamber in the energetic part of ECS, typically, the following types of electric energy conversion are used: the conversion of alternating voltage of power line to constant voltage, constant voltage regulation, and constant voltage conversion to alternating high voltage.

The conversion of the first type (from alternating voltage to constant voltage) is performed using uncontrolled rectifiers $(\mathrm{R})$ or controlled rectifiers (CR). Capacitor filters (F) are commonly used in order to smooth the surge control of voltage capacitor. When using uncontrolled rectifier the regulation of rectified (constant) voltage is realized through pulse converter
(PC) with the frequency or length modulation and LC-filters (F). The voltage inverters (VI) are applied to convert constant voltage to alternating voltage with frequency of 1-2 that is higher than frequency of power line voltage. The increase of the output voltage inverter to the desired level is achieved by step-up transformer (ST).

The easiest way to compensate the effect of changes in the alternating voltage of power line on average magnitude of voltage $u_{\mathrm{PS}}$ and at the discharge chamber consists of introducing a feedback from the output voltage of the inverter (output voltage sensor $\left(\mathrm{S}_{\mathrm{OV}}\right)$ Fig. 2) in automatic control inverter. Whereas the signal $u_{\mathrm{C}}$ of control inverter will be formed in the informational part of ECS with the reference of the signal task $u_{\mathrm{S}}$ and feedback signal $u_{\mathrm{FB}}$ that come from the input voltage sensor $\left(S_{I V}\right)$. This approach allows to solve the problem, but excludes the possibility of regulating the amplitude of the alternating voltage $u_{\mathrm{A}}$, which does not allow to adjust $\mathrm{OC}$ in OOM at more than 1:50. It does not allow to implement the full range of methods of ozone therapy $[2,3]$.

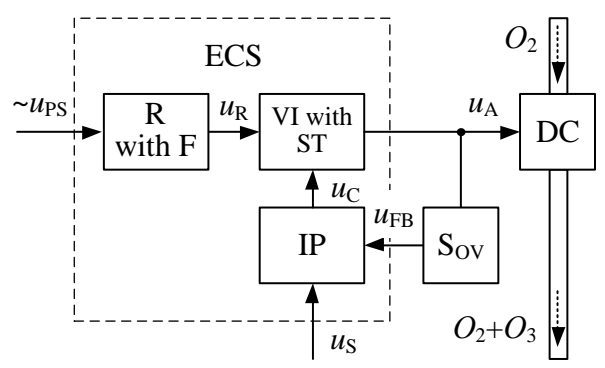

Fig. 2. Structure of ECS with output voltage feedback loop

The controlled rectifier with a filter ( $\mathrm{CR}$ with $\mathrm{F}$ ) is used to convert alternating voltage of power line to a constant voltage; the rectifier can have the regulation function of rectified voltage $u_{\mathrm{R}}$ (see Fig. 3). Here the control signal $u_{\mathrm{Cl}}$ of rectifier is formed in respect to the signal $u_{\mathrm{FB}}$ coming from the input voltages sensor $\left(\mathrm{S}_{\mathrm{IV}}\right)$ (for rectifier), that allows to compensate the effects of voltage changes $u_{\mathrm{PS}}$ at the input voltage inverter [6]. The control signal $u_{\mathrm{C} 2}$ of voltage inverter with step-up transformer (VI with ST) is formed with the voltage task $u_{\mathrm{S}}$ that allows you to get at the output of the inverter $u_{\mathrm{A}}$ voltage with frequencytime parameters, providing the target values of OC in OOM.

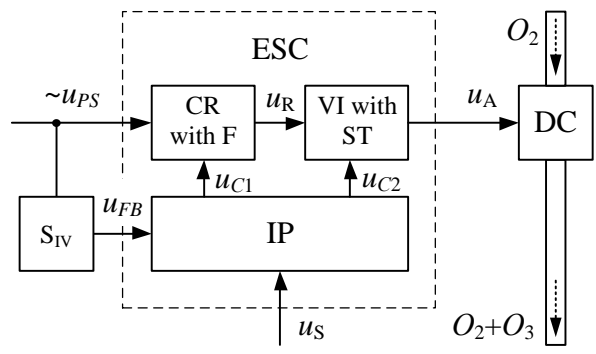

Fig. 3. Structure of ECS with input voltage sensor

However, with the need to regulate OC in OOM in the range 1:800, that is required for medical ozone generator, there is a need to control the rectifier not only to stabilized rectified voltage (i.e., compensate the impact of voltage instability of power line), but to change it in a certain range. This, in turn, 
leads to an increase in ripple ratio voltage at the input of the inverter by $20-40 \%$ as compared to the ECS, which is shown in Fig. 2. The presence of pulsations complicating management processes, and consequently, reduces the effectiveness of ECS to achieve a given concentration of ozone in the OOM. Providing acceptable ripple ratio voltage $(r \leq 10 \%)$, by reducing the range of the output voltage of the rectifier, control range of OC in OOM can increase only up to 1:100.

The control system with a pulse converter of constant voltage and filters circuit (PC with F) has larger possibilities to regulate and stabilize voltage $u_{\mathrm{RS}}$ on the discharge chamber, which is shown at Fig. 4. Here is information about the voltage changes of power line, and, consequently, the rectified voltage $u_{\mathrm{R}}$, information enters the information part as the signal $u_{\mathrm{FB}}$ from the input voltage sensor $\left(\mathrm{S}_{\mathrm{IV}}\right)$ (for pulse converter) [7]. The formation of the control signal $u_{\mathrm{C} 1}$ with the reference to the setting signal $u_{\mathrm{S}}$ and the feedback signal $u_{\mathrm{FB}}$ at the input voltage allows you to regulate the output voltage pulse converter $u_{\mathrm{P}}$ and ensure that it is invariant to input voltage changes $u_{\mathrm{R}}$. With ECS (Fig. 4) could be regulation of OC in OOM between 1:800 and more.

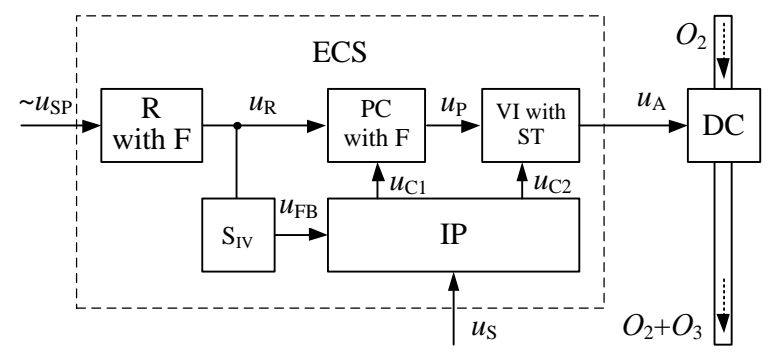

Fig. 4. Structure of ECS with pulse converter

The influence of such factors as OOM temperature can be significantly reduced, while ensuring the efficient cooling mode of the discharge chamber. OOM pressure changes are so insignificant that no essential changes occur in $\mathrm{OC}$ when those changes occur with the real values of the external load resistance of ozonator, especially with the use of degassers.

Thus, the main task of the electrical control system can be defined as the regulation and stabilization of amplitudefrequency-time parameters of the voltage on the discharge chamber, which can be achieved through the introduction of appropriate voltage feedback.

\section{PNEUMATIC CONTROL SYSTEM}

OOM flow through the ozonator in the general case can be defined by the expression

$$
Q=k \cdot S^{2} \Delta P / L
$$

where $k$ - coefficient of proportionality; $S$ - cross-sectional area of pneumatic ozonator tract; $\Delta P$ - pressure drop at the edges of pneumatic tract; $L$ - length of pneumatic tract.

From the above expression follows that to change the flow of OOM through pneumatic ozonator tract is possible with the help of two options. The first option is by changing the cross sectional area of pneumatic tract, the second option is due to changes in oxygen pressure at the input of DC. To solve such problems in pneumatic control systems pressure regulators are typically used to regulate and stabilize the gas pressure at its output, faucet, valves and chokes, which provide crosssectional change in pneumatic tract.

The scheme of the pneumatic system that implements the first control version is shown in Fig. 5. Here oxygen from an oxygen cylinder (OC) via a gear $(\mathrm{G})$ transferred to the input of the pneumatic control system (PCS). In this system executive part is in the form of the faucet $(F)$, which allows you to manually regulate the cross sectional area of pneumatic tract of ozonator and informational part is in the form of a flow meter (FM). Provided that the oxygen pressure at the output gear will not change, the system will provide a given flow rate of OOM through the discharge chamber (DC) for the load with some constant pneumatic-resistance, which will determine the value of the pressure at the output of pneumatic tract of ozonator.

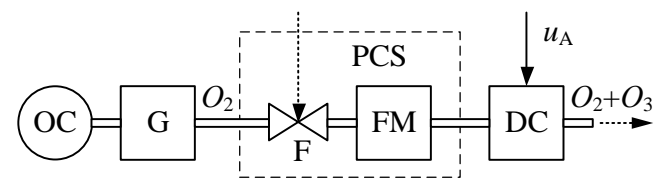

Fig. 5. Structure of PCS with manually regulation of the flow

However, changing the method of using ozone (eg, in the transition from ozonation of saline to fumigation of limb in a plastic bag) pneumatic resistance tract can vary by 4-5 times which increases the output pressure ozonator to $0.7-1$ bar, therefore, to reduce the flow of OOM by $30-50 \%$.

The solution of the regulation task and stabilization of the oxygen pressure at the input of the discharge chamber is possible through by performing in the form of IP an automatic pressure adjustment (APA) with a feedback (Fig. 6).

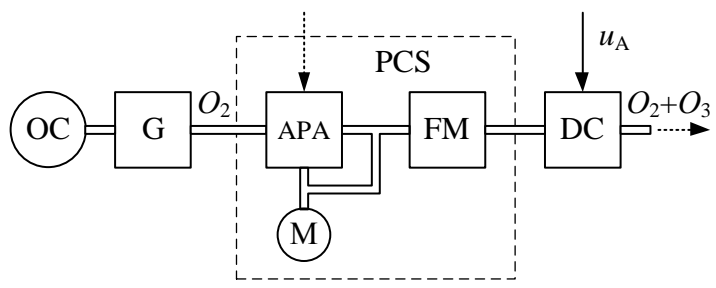

Fig. 6. Structure of PCS with automatic pressure adjustment

The velocity of OOM is set manually and monitored by FM. Manometer (M) is here an auxiliary tool for visual monitoring of oxygen pressure with its regulation in the manual mode.

The considered variants of PCS (Fig. 5-6) are quite viable. Some ozone generators (apparatus for the gas ozone therapy "KVAZAR", OC of the measuring devices of "KVAZAR", Nizhny Novgorod, synthesizer device of gas OOM a-s-GOKSF 5-02-OZONE, OJSC "LEPSE" Kirov and others) are applied with such systems to this day. However, current trends of medical ozonizer-construction are aimed at the automation of all processes in the ozonator, including the control flow process of OOM. 
The scheme of automatic flow control system is shown at Fig. 7 [8]. Here, the pressure regulator (PR) is switched on in consecutive ages with a pressure stabilizer (PS), which ensures the stability of the pressure drop at the edges of the pneumatic ozonator tract. Discrete regulation of the flow control of OOM according to binary principle is performed by throttle-valve block (TVB), which is controlled by the signal $u_{\mathrm{C}}$ from the informational part of OOM. Manual setting of regulator and pressure regulator, and also throttle-valve block is conducted at the manufacturer only during the system setup.

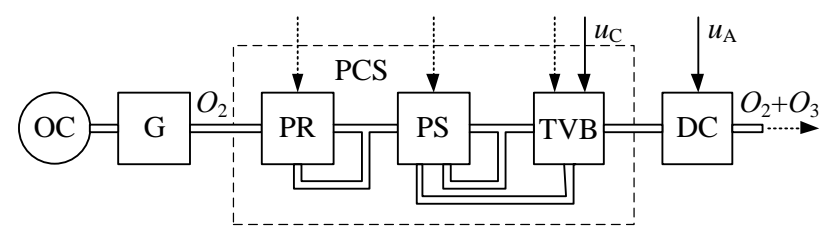

Fig. 7. Structure of PCS with throttle-valve block

In this case, by combining informational parts of pneumatic and electric systems, it is possible to set the flow rate of OOM and ensure its stabilization with an accuracy of $5 \%$, as in deviation of oxygen pressure, as at a of oxygen pressure at the inlet to $50 \%$ and load resistance change in the whole real range [9]. However, the mass-dimensional parameters of throttlevalve block leave much to be desired. That is why such PCS should be used only in cases where, strict dosing of OOM flow is necessary to stabilize all significant disturbing factors.

It is possible to improve the mass-dimensional parameters of PCS by using methods of pulse modulation to control the electromagnetic valve (EMV), which is installed in the pneumatic ozonator tract (Fig. 8). The control signal $u_{\mathrm{C}}$ of EMV is formed in the informational part of the electrical system control with the reference of signal task of OOM flow coming from the flow sensor, which is used as a differential pressure sensor $\left(S_{D P}\right)$. Receiver $(R e c)$ serves here to smooth the pressure fluctuation at the output of EMV and thus the assurance of flow uniformity of OOM.

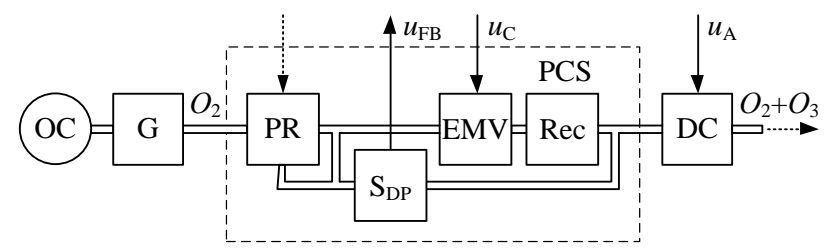

Fig. 8. Structure of PCS with pulse modulation

The tests of the system have shown that it is able to regulate and stabilize the flow of OOM with an accuracy of $10 \%$ when the oxygen pressure at the inlet to the ozonizer to $50 \%$ and change the air resistance throughout the whole range.

In any variant of the realization of the pneumatic control system, it is appropriate to use emergency release valve at the input of ozonator to limit the pressure at the input of PCS and force reset valve for oxygen venting from the pneumatic tract before disconnecting the ozonator from the source of oxygen. In addition, it is useful to apply a filter to clean the oxygen of solids and humidity [10].

\section{CONCLUSIONS}

In conclusion, it should be noted that in choosing of any construction variant of control systems by medical ozonator, is necessary to proceed from the conditions of relevancy and feasibility. During the universal ozonator development which is intended for research and development and new methods of ozone therapy, it should be provided a considerable range of opportunities for regulation and stabilization of OC in OOM and its flow rate. For this purpose the electric control system with a pulse transmitter is most suitable (see Fig. 4). Pneumatic system in this case is better to construct according to the third or fourth variants (Fig. 7 and 8, respectively). For more simple and repetitive manipulations in ozonators, it is better to use simpler technical solutions, such as the second or third variants of ECS (Figs. 2 and 3) and the second variant of PCS (Fig. 6). This approach will significantly reduce the cost of the ozonator. To regulate the $\mathrm{OC}$ in $\mathrm{OOM}$ it may be justified to use the first variant of ECS (Fig. 1), provided that the OC sensor will have good mass-dimensional parameters, adequate performance and reliability. It is also important, to our opinion, the production of specialized ozonators for physicians of various specialties, in order to avoid redundancy of technical solutions and thus, reduce the cost of products at high enough quality.

\section{REFERENCES}

[1] "Nizhny Novgorod Medical Journal. Appendix: Ozone therapy. - N. Novgorod, 2003, - 344 p. (In Russian)

[2] O. Maslennikov, K. N. Kontorshchikova "Practical ozone therapy: The Handbook. - N. Novgorod: "Vector-TiS", 2003, - 52 p. (In Russian)

[3] O.V. Maslennikov, K.N. Kontorschikova. "Ozone therapy: The Internal Diseases,"N. Novgorod: "Vektor-TiS”, 2003, - 132 p (In Russian)

[4] E.I. Sokol, A.V. Kipenskiy, A.A. Lashin. "The Specifics of Controlling the Concentration of Ozone in Medical Ozone Generators" // Proceedings of the Conference "New technologies and preformed natural healing factors." - Kharkov: HMPA, 2002. - 229-231 p. (In Russian)

[5] E.I. Sokol, A.V. Kipenskiy, A.A. Lashin. "The compensation of the perturbations in the regulation of the parameters of the ozone-oxygen mixture in medical ozone generators // Nizhny Novgorod Medical Journal. - Nizhny Novgorod, 2003. - 313-315 p. (In Russian)

[6] A.V. Kipenskiy, E.I. Sokol, V.I. Ryabenkiy and others. Patent № 23060 UA, MKV N 02 M 7/12. "The method of the direct microprocessor control of the semiconductor power converter and device for its implementation” № 94043468; declared 11.05.94; published 30.06.98, bulletin № 3. (In Ukrainian)

[7] A.V. Kipenskiy, A.V. Kipenskaya. Patent № 1742964 RF, MKI 5 NO2 M 7/12. The method of controlling of the semiconductor converters DC voltage / № 4762580/07; declared 27.11.89; published 23.06.92, bulletin № 23. (In Russian)

[8] E.I. Sokol, A.V. Kipenskiy, A.A. Lashin and others. "The combined system of control the consumption of ozone-oxygen mixture in medical ozone generators" // International Medical Journal. Appendix: "Ozone therapy." - Kharkov, 2003. - 158-161p. (In Russian)

[9] A.V. Kipenskiy, E.I. Sokol, A.A. Lashin and others. "The characteristics and results of operation of the medical ozone OM 80/1" General Reanimatology. - M.: Research Institute of General Reanimatology, of the Russian Academy of Medical Sciences, 2006. - T. 2. - № 4/1. - 297 301p. (In Russian)

[10] A.V. Kipenskiy, E.I. Sokol, A.A. Lashin and others. "The development of medical ozone OM80/1 and the results of its tests" Herald of Physiotherapy and Health Resort. - Evpatoria: EIRITS, 2006. - № 5. 76-78p. (In Russian) 